\title{
Does Childhood Executive Function Predict Adolescent Functional Outcomes in Girls with ADHD?
}

\author{
Meghan Miller • Stephen P. Hinshaw
}

Published online: 4 December 2009

(C) The Author(s) 2009. This article is published with open access at Springerlink.com

\begin{abstract}
We prospectively followed an ethnically and socioeconomically diverse sample of preadolescent girls with ADHD $(n=140)$ and matched comparison girls $(n=88)$ over a period of 5 years, from middle childhood through early/mid-adolescence. Our aim was to examine the ability of measures of childhood executive function (EF) to predict functional outcomes in adolescence. Measures of neuropsychological functioning comprised the childhood predictors, with academic, social, and global functioning serving as adolescent criterion measures. Results indicated that childhood EF predicted (a) academic achievement and social functioning across our entire sample (independent of diagnostic group status) and (b) global functioning only in girls with ADHD (independent of IQ). These results highlight the non-specificity of EF deficits and suggest the importance of assessing and developing interventions that target EF impairments, particularly in those at high-risk for negative outcomes, in order to prevent long-term difficulties across a range of important functional domains.
\end{abstract}

Keywords Attention-deficit/hyperactivity disorder (ADHD) - Executive function · Neuropsychology ·

Functional skills $\cdot$ Females

A considerable body of literature reveals that neuropsychological deficits characterize a subset of individuals with attention-deficit/hyperactivity disorder (ADHD). One aspect of these deficits involves executive function (EF), an umbrella term that refers to neuropsychological abilities

M. Miller $(\bowtie) \cdot$ S. P. Hinshaw

Department of Psychology, University of California, Berkeley,

Tolman Hall \#1650,

Berkeley, CA 94720-1650, USA

e-mail: meghanmiller@berkeley.edu such as planning, organization, reasoning, response inhibition, decision-making, working memory, and set-shifting (Pennington and Ozonoff 1996; Barkley 1997; Tranel et al. 1994; Osmon 1996) - abilities that are mediated by the prefrontal cortex (PFC) and its extensive interconnections with other brain regions (Tranel et al. 1994). Neuroimaging studies have shown that deficits in EF in children with ADHD may be related to structural and functional differences in regions including the PFC and frontostriatal pathways (for a review, see Seidman et al. 2005b), although there is considerable debate regarding (a) the nature and causes of EF deficits in individuals with ADHD and (b) the validity of the EF construct, including exactly which abilities fall into this domain (see Jurado and Rosselli 2007).

Multiple theories have attempted to characterize the nature of EF deficits in individuals with ADHD. Many of these models pinpoint dysfunction in the PFC as being central to the development of EF deficits in ADHD and/or symptoms related to the ADHD syndrome (Barkley 1997; Benson 1991; Pennington and Ozonoff 1996). Others have posited that ADHD results from noncortical dysfunction but that prefrontally-mediated mechanisms account for symptom reduction and potential improvements in EF throughout development (Halperin and Schulz 2006). Regardless of the mechanisms by which EF deficits develop and/or improve over time, having intact EFs appears important for success across multiple domains. Still, surprisingly little is known about the predictive relationships between EF and functional outcomes, particularly in individuals who are at high risk for EF deficits, such as those with ADHD.

In addition to ADHD, EF deficits have been found in several neurodevelopmental disorders such as autism, conduct disorder, and Tourette syndrome (Barkley 1997; 
Pennington and Ozonoff 1996; Willcutt et al. 2005). Thus, the suggestion is that these deficits constitute a transdiagnostic mechanism. Another issue is that EF deficits are not found in all individuals with these conditions (including ADHD) but may instead characterize subgroups of these populations. The present focus is on EF deficits in girls with ADHD - deficits that appear to persist at least until adolescence (Fischer et al. 2005; Hinshaw et al. 2007) and the longitudinal associations of these deficits with a range of functional outcomes.

Although many recent investigations have shown that EF deficits are salient in at least a subgroup of children with ADHD (Coghill et al. 2005; Hinshaw et al. 2002; Willcutt et al. 2005; Nigg et al. 2002; Seidman et al. 2005a, 2006), not enough is known about the associations between these deficits and the emergence of "real world" functional impairments, such as in academic and social abilities (but see Biederman et al. 2004 and Diamantopoulou et al. 2007 for exemplary research in this regard), particularly not in girls. As a result, ascertaining longitudinal linkages between EF deficits and functional impairments is of central importance for at least two reasons: (a) to develop deeper understanding of mechanisms underlying the ADHD syndrome as well as the EF construct; and (b) to create preventive and intervention strategies for those with ADHD, which might involve the targeting of EF deficits. Additionally, it is essential to gain an understanding of these associations in girls with ADHD, given the relative paucity of research on females with this condition (Hinshaw and Blachman 2005).

EFs have been linked to the successful performance of a wide range of tasks, including academic performance as well as social/interpersonal skills (Biederman et al. 2004; Clark et al. 2002; McEvoy et al. 1992). Focusing first on academic achievement, the abilities to organize, plan, reason, and keep tasks in working memory are essential to school performance. In fact, it has been shown that children with ADHD who exhibit EF deficits are likely to require intensive academic intervention in order to prevent academic failure or drop out of school (Barry et al. 2002; Biederman et al. 2004; Massetti et al. 2007). Yet much of this literature is based on studies of boys with ADHD; a clear gap is apparent regarding girls with this condition. Thus, a key aim of the present investigation is to determine predictive associations between baseline EF variables and adolescent academic achievement in a female sample.

Less is known about the relationship between EF deficits and difficulties in interpersonal relationships in girls with ADHD. Yet inhibiting certain behaviors and shifting mental sets appear crucial for interpersonal relationships. Some studies have found EFs to be linked to social abilities (Clark et al. 2002; Diamantopoulou et al. 2007), whereas others have not (Biederman et al. 2004), and very little research has focused on these associations in girls with ADHD (for an exception, see Diamantopoulou et al. 2007). Additionally, frontal lesions can be implicated in poor social interactions, as is illustrated by the well-known case of Phineas Gage, who faced extreme social difficulties after suffering frontal lobe damage (Harlow 1848). Some research has suggested that EF facilitates children's theory of mind (ToM) (Hughes and Ensor 2007), an ability that is crucial to successful social interactions. Overall, because academic success and strong interpersonal relationships are two crucial components of healthy adjustment, understanding the specific predictive associations between EF and these two areas of functioning is a priority.

Global functioning is also a crucial outcome measure. Findings are limited and mixed in terms of the associations between EF deficits and global impairments in ADHD. One study did not find such linkages (Biederman et al. 2004), although sex differences were not assessed. Determining childhood predictors of later global functioning (which, by definition, involves cross-domain measures) in girls is a particularly important area of research, because global functioning is closely linked to overall success in meeting key developmental transitions in adolescence. Indeed, understanding the associations between childhood EF and later global functioning could prove especially important in determining who may be at risk for becoming generally maladjusted in adolescence or beyond.

With an emphasis on clinical relevance and clinical significance related to global functioning, Owens et al. (2009) demonstrated a means by which to identify competent groups of children among those at risk for poor developmental outcomes - specifically, youth with ADHD - by evaluating the presence of positive adjustment (PA) in adolescence across six domains: ADHD symptoms, externalizing symptoms, internalizing symptoms, social skills, peer acceptance, and school achievement. They also calculated a classification of overall PA in the present sample, defined as performing within the normal range of the typical adolescent across the majority of these domains (see Method for additional details and see also Lee et al. 2008). A key aim of the present report is to predict, from neuropsychological and EF variables measured in childhood, which youth are positively adjusted in adolescence. We measure PA in three specific domains of special relevance to ADHD-academic achievement, peer acceptance, and social skills.

Previous studies from the present sample have documented that in childhood as well as in adolescence the ADHD group showed clear EF impairments relative to the comparison group, even when controlling for key demographic variables and comorbidities (Hinshaw et al. 2002; Hinshaw et al. 2007). Yet no current work has examined the ability of EF measures in childhood to predict functional 
outcomes in adolescence prospectively. Thus, we address the following question: Does childhood EF predict adolescent academic achievement (including academic achievement PA), social functioning (including social skills and peer acceptance PA), and global functioning in a female sample? We hypothesize that poorer performance on tasks of EF in childhood will predict worse outcomes in adolescence across all of these domains, in girls with and without ADHD, but that these relationships will be more pronounced in girls with ADHD than in the comparison sample. In other words, ADHD status will serve as a moderator of EF-functional outcome predictions. We make this latter prediction because of the strong likelihood that the symptomatology of ADHD will combine with EF deficits to yield particularly problematic adolescent outcomes. We also include key covariates to ascertain the specificity of any EF-functional outcome associations.

\section{Method}

\section{Overview of Procedure}

We utilized data from an ongoing longitudinal study of behavioral, neuropsychological, social, and family functioning in 228 girls, 140 with rigorously diagnosed childhood ADHD and 88 matched comparison girls. All participated in summer research programs and extensive testing during childhood (baseline: ages 6-12), and they were followed prospectively into adolescence, completing extensive evaluation again 5 years later (see Hinshaw 2002; Hinshaw et al. 2006).

During the baseline summer programs, we emphasized multi-domain evaluation of key areas of relevance to childhood functioning, and we performed an extensive neuropsychological battery including $\mathrm{EF}$, language, and motor speed measures. Hinshaw (2002) provides details on summer programs and the multiple-gating procedure used for screening and diagnostic assessment; Hinshaw et al. (2002) present extensive data on the baseline neuropsychological battery, administered when any medicated girls were not receiving stimulant medication. Well-trained graduate students and bachelor's-level research assistants, all of whom were closely supervised, administered the neuropsychological tests (Hinshaw et al. 2002).

For the 5-year follow up assessments, 209 out of 228 girls were retained $(92 \%)$ for extensive individual and family evaluation. This level of participant retention was a function of considerable efforts to contact families who had moved or relocated. As indicated in Hinshaw et al. (2006), the 209 girls in the follow-up sample and the 19 lost to attrition were statistically indistinguishable with respect to nearly all baseline variables examined. The key goal was to appraise, via multi-informant and multi-method procedures, levels of symptomatology and adjustment/impairment in key domains of psychiatric, academic, and social functioning. Hinshaw et al. (2006) provide extensive details about these follow-up assessments, which had received full approval from the Committee for the Protection of Human Subjects.

\section{Participants}

The complete sample consists of 93 girls with ADHDCombined type (ADHD-C), 47 with ADHD-Inattentive type (ADHD-I), and 88 comparison girls (see Hinshaw 2002 for full details). The comparison sample was matched, at a group level, with the clinical group in terms of both age and ethnicity. This overall sample is both socioeconomically and ethnically diverse (family incomes ranging from public assistance to upper-middle class; 53\% White, 27\% AfricanAmerican, 11\% Latina, 9\% Asian-American). At baseline (summers of 1997, 1998, or 1999), these 228 girls were 6 to 12 years of age $(M=9.6$ years). At follow up, the 209 retained girls were 11 to 18 years of age ( $M=14.2$ years).

In terms of comorbidity at baseline, the percentage of girls who met criteria for reading disorder was $11.1 \%$ for ADHD-C, $14.9 \%$ for ADHD-I, and $4.5 \%$ for the comparison group. The percentage of girls who met criteria for conduct disorder (CD) based on parent report from the Diagnostic Interview Schedule for Children (4th ed., DISCIV; Shaffer et al. 2000) was $26.9 \%$ for ADHD-C, $10.6 \%$ for ADHD-I, and $0 \%$ for the comparison group. For oppositional defiant disorder (ODD), the percentages were $71 \%$ for ADHD-C, $46.8 \%$ for ADHD-I, and 6.8\% for the comparison group. We note that staff at the summer camps noted lower rates of CD- and ODD-related behaviors via ratings and direct observations than had been the case for previous programs for boys with ADHD; it may well be that parents of girls with ADHD witness considerable oppositionality at home with their daughters.

Approximately $52 \%$ of the girls with ADHD had received psychotropic medication (typically stimulants) during the 5-year follow-up interval, but receipt of medication did not influence any core outcome measures (Hinshaw et al. 2006). The vast majority of the girls with ADHD (but only about $11 \%$ of the comparison girls) had received psychosocial services or school-based services during the follow-up interval. In terms of change in diagnostic status, the majority of comparison girls maintained their non-ADHD status at follow-up (77/81). There was a greater persistence of ADHD-I than ADHD-C, with 26/41 of the ADHD-I girls maintaining the ADHD-I status at follow-up but only $33 / 85$ of the ADHD-C group maintained such status. Of the 20 who lost the ADHD-I diagnosis in adolescence, 15 were classified as comparisons, and 5 as ADHD-C/HI. Of the 52 who lost the ADHD-C diagnosis in adolescence, 29 were 
classified as comparisons, 20 as ADHD-I, and three as ADHD-HI. We classified participants by baseline diagnostic status for our analyses, as our overall objective was to predict adolescent functional outcomes from childhood diagnostic status and neuropsychological variables.

\section{Baseline Measures}

We selected several well-established and validated measures of EF from the baseline neuropsychological battery. Selection was based on (a) their conceptual relevance to the EF construct and (b) prior research revealing that these measures were those that best differentiated our youth with ADHD from comparison youth, even with statistical control of demographics and comorbidities. All measures were adjusted such that higher scores equate to better performance, for ease of interpretation of findings.

Rey Osterrieth Complex Figure (ROCF; Osterrieth 1944) The ROCF is a complex cognitive task that requires an individual to copy and later recall a complex figure composed of 64 segments. We analyze the Copy condition of this task (participants draw the figure with no delay), which taps multiple domains of EF, such as planning, working memory, inhibitory control, attention to detail, and organization. Only the Copy condition differentiated the girls with ADHD from our comparison sample at baseline (Sami et al. 2003).

The ROCF has been successfully used to distinguish patients with frontal lobe lesions from those without (Lezak 1995), as well as children with ADHD from those without (Carte et al. 1996; Nigg et al. 1998; Sami et al. 2003).

Scores from various methods of scoring the ROCF are significantly correlated with other measures of EF (Troyer and Wishart 1997; Somerville et al. 2000; Watanabe et al. 2005), indicating that the ROCF is indeed assessing one or more aspects of EF. We used two validated methods of scoring the ROCF: the Error Proportion Score (EPS) developed by Sami et al. (2003), and the organization score from the Developmental Scoring System by Waber and Holmes (1985). The EPS is a ratio (number of errors/total number of segments drawn) and is a measure of efficiency (Sami et al. 2003). The organization score consists of a score from 1 to 13 and represents five developmental levels ranging from poor to excellent (Waber and Holmes 1985). The intraclass correlation between pairs of the three primary scorers for the EPS ranged from 0.91 to 0.94 (drawings $n=84-195$ across rater pairs). For the organization system, the intraclass correlation between the two primary scorers was $0.90(n=139)$. Among all of the EF measures in our battery, the EPS from the ROCF showed the largest effect size $(d=0.90)$ in differentiating the girls from ADHD from the comparison sample during childhood (Hinshaw et al. 2002; Sami et al. 2003).
Conners' Continuous Performance Task (CPT; Conners 1995) The CPT is a computerized visual task of attentional processing and response inhibition that requires the participant to press the spacebar when target letters appears on the screen (all letters except ' $\mathrm{X}$ '), and not respond to the letter ' $\mathrm{X}$ '. The 14-minute task consists of trials that are presented in six blocks (interstimulus intervals: $1 \mathrm{~s}, 2 \mathrm{~s}$, and $4 \mathrm{~s}$ ); stimuli are displayed for $250 \mathrm{~ms}$. This task differs from other commonly-used continuous performance tasks by featuring frequent display of target stimuli (requiring response) and relatively infrequent display of non-targets (requiring nonresponse), so that response inhibition rather than detection of rare stimuli is featured. A recent neuroimaging study suggested the involvement of a neural network including the frontal, cingulate, parietal, occipital, and temporal regions as well as the basal ganglia and cerebellum in the performance of this task (Ogg et al. 2008). We utilized two scores: the percentage of omission errors and the percentage of commission errors. Again, these variables were reverse-scored such that higher scores equate to better performance. Our prior research has shown significant differences in both omission and commission errors between ADHD and comparison girls in the present sample (at baseline and follow-up), whereby the girls with ADHD reveal higher percentages of both types of errors, with effect sizes in the medium range (Hinshaw et al. 2002; Hinshaw et al. 2007). Conners (1995) provided criterionrelated validity data for omission and commission errors based on known-groups differentiation.

Cancel Underline (CUL) The CUL is a modified version of the Underlining Task (Rourke and Orr 1977). It measures the EF component of inhibitory control and rapid, accurate visual discrimination, abilities that recruit anterior cingulate and prefrontal regions (Cabeza and Nyberg 1997). Participants were instructed to underline targets (shape or consonant sequences) and cancel out nontargets (ratio of 1:5). Our analysis is based on correct minus incorrect responses (Nigg et al. 1998). Previous research has shown medium-sized differences between ADHD and comparison individuals on this measure, with those with ADHD performing worse (Carte et al. 1996; Hinshaw et al. 2002, 2007; Nigg et al. 1998).

\section{Follow-up Measures}

The following measures of functional skills at our 5-year follow-up were chosen from an extensive battery because they reflected the outcomes of interest (academics, social and peer relations, global impairment) as well as a variety of data collection methods, including objective testing as well as parent and teacher reports.

Wechsler Individual Achievement Test (WIAT; Wechsler 1992) The WIAT is a psychometrically sound assessment 
of academic achievement, with both internal consistency and test-retest reliability estimates above 0.85 for most composite scores (Wechsler 1992). Both the Basic Reading and Math Reasoning composite scores were used as measures of academic functioning at follow-up.

Dishion Social Preference Scale (DSPS; Dishion 1990) The DSPS is a 3-item teacher-completed scale that measures proportion of peers who accept, reject, and ignore the adolescent in question on a scale of $1-5$. We subtracted "rejected" from "accepted" ratings to obtain a widely-used social preference score (see Lahey et al. 2004; Sandstrom and Cillessen 2003). Although the "gold standard" for appraising peer preference is sociometric appraisals directly from agemates, obtaining schoolwide peer nominations for a middle-school and high-school sample was prohibitive. Furthermore, major concerns exist regarding the accuracy of self-reports from individuals with ADHD (e.g., Barkley 2006), so we ruled out self-reported appraisals of peer status. Dishion (1990) provided data on the ability of the DSPS to provide a valid approximation to peer sociometric measures, having found moderately strong correlations between items of the DSPS and peer-derived sociometric data. The DSPS is frequently used to estimate peer regard in middle-school and high-school samples.

Columbia Impairment Scale (CIS; Bird et al. 1993) The CIS is a 13-item parent-reported scale that assesses functioning across the domains of interpersonal relations, broad psychopathological domains, school/job functioning, and use of leisure time. Parents rate their child's impairment for each item using a 5-point scale, yielding an index of global impairment. We reverse-scored the measure so that higher scores equate to less impairment. The CIS has been used extensively in investigations of child and adolescent psychopathology and treatment response (e.g., MTA Cooperative Group 1999).

Positive adjustment (PA) Owens et al. (2009) and Lee et al. (2008) developed PA variables across six domains. Owens et al. (2009) chose cut-offs within each domain that corresponded to the level at which a child would be viewed as not requiring treatment for difficulties in that area. An adolescent was considered to be positively adjusted in a respective domain if she scored (a) at or below the midpoint between the ADHD and comparison means on symptom measures and negative social preference measures, (b) at or above the midpoint of the ADHD and comparison means on the social skills measure, and (c) greater than or equal to 85 (1 SD below national norms) on both math and reading academic achievement measures. Overall PA rates were determined by appraising percentages of girls who met domain-specific PA criteria for all or nearly all of the six relevant domains. Based on the functional outcomes of interest to our analyses (i.e., academic, social, global), only the following were examined in this investigation: academic achievement, social skills, and peer acceptance PA. These variables were dummy coded such that $0=$ does not meet PA criteria and 1=meets PA criteria in each PA category. See Owens et al. (2009) for a more detailed description of the determination of PA.

\section{Covariates}

Two key covariates were utilized separately in the data analyses. First, to indicate intelligence, we entered the Full Scale IQ (FSIQ) score from the Wechsler Intelligence Scale for Children-Third Edition (WISC-III; Wechsler 1991), which was administered in total to all participants at baseline. Second, to determine whether EF measures provided greater prediction to our adolescent criterion measures for girls with ADHD than comparison girls, we entered the baseline variable of group status (ADHD vs. comparison, dummy coded as 1 vs. 0 ). Because of significantly reduced statistical power, differences in ADHD-C vs. ADHD-I were not examined. We note that exploratory analyses suggest that this subtype differentiation yielded no predictive power with respect to any criterion measure. Furthermore, our past research has revealed no significant ADHD-C vs. ADHD-I differences on most correlates in our extensive database (Hinshaw 2002; Hinshaw et al. 2006).

\section{Data Analytic Plan}

All statistical analyses were performed with SPSS for Macintosh, Version 16. After carefully inspecting data for out-of-range values and pulling 6 outliers in to within $3 S D$ of the mean (all within the CPT omissions variable), our initial step was to perform Pearson correlations (for continuous variables) or $t$-tests (for dichotomous PA variables) regarding the associations between baseline EF variables and follow-up criterion variables. Following a significant correlation or $t$-test, we used linear or (in the case of PA variables) binary logistic regression with EF measures as predictors and academic, social, and global impairment measures as criterion variables, first controlling for IQ and then controlling for group status (ADHD vs. comparison), both of which were assessed at baseline. On the basis of these initial regressions, we reasoned that because IQ deficits are inherent to ADHD, controlling for both IQ and group concurrently would constitute overcontrol (see Miller and Chapman 2001). Thus, we emphasize findings with the more stringent covariate of group status (ADHD vs. comparison). Finally, following a significant regression analysis, we added the interaction term of the EF predictor $\mathrm{x}$ group status as the last step in the equation, to test for moderation by ADHD status. 
To maintain clinical relevance, we conducted regression analyses using single predictor variables.

\section{Results}

Initial correlations revealed statistically significant associations between several predictor-criterion pairs (Table 1). Only one of the EF variables (ROCF EPS) was significantly correlated with follow-up WIAT Reading scores. Both ROCF measures were significantly correlated with followup WIAT Math scores. Overall, associations between EF measures and academic, social, or global impairment scores were small, with the ROCF EPS variable yielding small- to medium-sized associations (i.e., in the 0.3 range; see Cohen 1988). Initial $t$-tests also revealed statistically significant associations between several baseline EF variables and follow-up PA variables. See Table 2 for these results.

Results from the linear regression analyses revealed significant predictive associations, when controlling for IQ, between (a) baseline ROCF EPS and follow-up WIAT Math, DSPS, and CIS; (b) baseline CPT omissions and follow-up DSPS; and (c) baseline CPT commissions and follow-up DSPS (see Table 3). When controlling for diagnostic group, only the predictive associations between (a) baseline ROCF EPS and follow-up WIAT Math, (b) baseline CPT omissions and follow-up DSPS, and (c) baseline CPT commissions and follow-up DSPS remained statistically significant (see Table 3), indicating that baseline EF variables predicted adolescent functional outcomes in the domains of academic achievement and social preference when controlling for diagnostic group. We therefore selected diagnostic group as the covariate for all subsequent analyses. With such control, the ROCF organization score did not show predictive associations with any of the criterion measures in the linear regression analyses.

Binary logistic regression analyses are displayed in Table 4. When controlling for IQ, these analyses indicate significant predictive associations between (a) baseline ROCF EPS and follow-up peer acceptance PA; (b) baseline CPT omissions and follow-up peer acceptance PA, academic achievement PA, and social skills PA; and (c) baseline CPT commissions and follow-up peer acceptance PA. When controlling for group status, only the associations between (a) baseline ROCF EPS and follow-up peer acceptance PA; (b) baseline CPT omissions and follow-up peer acceptance PA and academic achievement PA; and (c) baseline CPT commissions and follow-up peer acceptance PA remained statistically significant. Thus, when controlling for group status (the more stringent covariate), baseline EF variables predicted adolescent PA in peer acceptance and academic achievement, consistent with our linear regression analyses. In all cases of significant predictive associations, better EF scores predicted better adolescent status.

Moderator analyses revealed that group status did not moderate the relationships between any of the following baseline variables and follow-up WIAT Math scores: ROCF Organization $(p=0.258)$ or ROCF EPS $(p=0.278)$. Group status also did not moderate the relationship between baseline ROCF EPS and follow-up WIAT Reading scores $(p=0.388)$, nor did it moderate associations between baseline ROCF EPS $(p=0.360)$, baseline CPT omissions $(p=0.957)$, or baseline CPT commissions $(p=0.459)$ and follow-up DSPS scores. Group status did not moderate associations between any of the following baseline variables and followup CIS scores: ROCF EPS ( $p=0.097)$, CPT omissions $(p=$ 0.076 ), or CPT commissions ( $p=0.798)$. In terms of followup PA variables, group status did not moderate associations between any of the baseline EF variables and follow-up peer acceptance PA: ROCF organization $(p=0.769)$, ROCF EPS ( $p=0.735)$, CPT omissions ( $p=0.334)$, or CPT commissions $(p=0.623)$, nor did it moderate the associations between any of the following baseline EF variables and follow-up academic achievement PA: ROCF EPS $(p=0.937)$, CPT omissions, $(p=0.524)$, or CPT commissions $(p=0.732)$. We note that we also performed exploratory analyses controlling

Table 1 Correlations Between Baseline EF Variables and Follow-up Outcome Variables

\begin{tabular}{|c|c|c|c|c|c|c|c|c|}
\hline & \multicolumn{2}{|c|}{$\begin{array}{l}\text { Follow-up WIAT Math } \\
d f=194-199\end{array}$} & \multicolumn{2}{|c|}{$\begin{array}{l}\text { Follow-up WIAT Reading } \\
d f=194-199\end{array}$} & \multicolumn{2}{|c|}{$\begin{array}{l}\text { Follow-up DSPS } \\
d f=144-148\end{array}$} & \multicolumn{2}{|c|}{$\begin{array}{l}\text { Follow-up CIS } \\
d f=198-203\end{array}$} \\
\hline & $r$ & $p$ & $r$ & $p$ & $r$ & $p$ & $r$ & $p$ \\
\hline Baseline ROCF organization & 0.20 & $0.003 * *$ & 0.08 & 0.121 & 0.15 & $0.038^{*}$ & 0.11 & 0.061 \\
\hline Baseline ROCF EPS & 0.32 & $0.000 * *$ & 0.20 & $0.002 * *$ & 0.23 & $0.003 * *$ & 0.29 & $0.000^{* *}$ \\
\hline Baseline CPT omissions & 0.05 & 0.237 & -0.01 & 0.460 & 0.22 & $0.004 * *$ & 0.18 & $0.007 * *$ \\
\hline Baseline CPT commissions & 0.01 & 0.425 & -0.02 & 0.407 & 0.17 & $0.020^{*}$ & 0.08 & 0.134 \\
\hline Baseline CUL & 0.11 & 0.056 & 0.08 & 0.131 & 0.16 & $0.030 *$ & 0.13 & $0.037^{*}$ \\
\hline
\end{tabular}

DSPS Dishion Social Preference Scale, WIAT Wechsler Individual Achievement Test, CIS Columbia Impairment Scale, ROCF Rey-Osterrieth Complex Figure (EPS error proportion Score), CPT Continuous Performance Test, CUL Cancel Underline

$* p<0.05 ; * * p<0.01$ 
Table 2 T-tests for Baseline EF Variables and Follow-up PA Outcome Variables

\begin{tabular}{|c|c|c|c|c|c|c|}
\hline & \multicolumn{2}{|c|}{$\begin{array}{l}\text { Follow-up Social Skills PA } \\
d f=197-202\end{array}$} & \multicolumn{2}{|c|}{$\begin{array}{l}\text { Follow-up Peer Acceptance PA } \\
d f=197-202\end{array}$} & \multicolumn{2}{|c|}{$\begin{array}{l}\text { Follow-up Academic Achievement PA } \\
d f=194-199\end{array}$} \\
\hline & $t$ & $p$ & $t$ & $p$ & $t$ & $p$ \\
\hline Baseline ROCF organization & -1.86 & 0.065 & -2.30 & $0.023 *$ & -1.11 & 0.268 \\
\hline Baseline ROCF EPS & -2.15 & $0.033^{*}$ & -4.10 & $0.000 * *$ & -2.71 & $0.009 * *$ \\
\hline Baseline CPT omissions & -2.59 & $0.011^{*}$ & -3.02 & $0.003 * *$ & 2.14 & $0.034 *$ \\
\hline Baseline CPT commissions & 0.22 & 0.827 & -2.16 & $0.032 *$ & -0.32 & 0.752 \\
\hline Baseline CUL & -0.67 & 0.506 & -1.76 & 0.080 & -1.60 & 0.112 \\
\hline
\end{tabular}

$P A$ positive adjustment, ROCF Rey Osterrieth Complex Figure (EPS error proportion score), CPT Continuous Performance Task, CUL Cancel Underline

${ }^{*} p<0.05 ; * p<0.01$.

for comorbid ODD/CD and results remained largely the same with two exceptions: baseline ROCF EPS significantly predicted follow-up DSPS scores and CIS scores (previously marginally significant associations), and group status still did not moderate either of these associations.

Although none of the EF $\times$ group interaction terms was significant, there were some marginally significant findings, and interaction tests of moderator variables typically lack statistical power (McClelland and Judd 1993). Thus, we performed exploratory regression analyses separately in each group (ADHD vs. comparison) for those $\mathrm{EF}$ variables that had yielded statistically significant associations with criterion measures. It should be noted that, due to lack of variance in the comparison group on the binary PA measures, we focus only on exploratory analyses between baseline EF variables and continuous follow-up measures. When controlling for

Table 3 Linear Regression Analyses for Baseline EF Variables and Follow-up Variables

\begin{tabular}{|c|c|c|c|c|c|c|c|}
\hline & Standardized $\beta$ & $\Delta R^{2}$ & Sig. & & Standardized $\beta$ & $\Delta R^{2}$ & Sig. \\
\hline \multicolumn{8}{|l|}{ WIAT Math Scores } \\
\hline Step 1: IQ & 0.73 & 0.53 & $0.000 * *$ & Step 1: Group & -0.52 & 0.27 & $0.000 * *$ \\
\hline Step 2: ROCF Organization & 0.08 & 0.01 & 0.121 & Step 2: ROCF Organization & 0.11 & 0.01 & 0.087 \\
\hline or Step 1: IQ & 0.73 & 0.53 & $0.000 * *$ & or Step 1: Group & -0.52 & 0.27 & $0.000 * *$ \\
\hline Step 2: ROCF EPS & 0.11 & 0.01 & $0.038^{*}$ & Step 2: ROCF EPS & 0.16 & 0.02 & $0.012^{*}$ \\
\hline \multicolumn{8}{|l|}{ WIAT Reading Scores } \\
\hline Step 1: IQ & 0.59 & 0.34 & $0.000 * *$ & Step 1: Group & -0.34 & 0.12 & $0.000^{* *}$ \\
\hline Step 2: ROCF EPS & -0.01 & 0.00 & 0.923 & Step 2: ROCF EPS & 0.07 & 0.00 & 0.326 \\
\hline \multicolumn{8}{|l|}{ Dishion Social Preference Scale } \\
\hline Step 1: IQ & 0.14 & 0.02 & 0.090 & Step 1: Group & -0.32 & 0.11 & $0.000 * *$ \\
\hline Step 2: ROCF EPS & 0.20 & 0.04 & $0.022^{*}$ & Step 2: ROCF EPS & 0.15 & 0.02 & 0.066 \\
\hline or Step 1: IQ & 0.14 & 0.02 & 0.098 & or Step 1: Group & -0.35 & 0.12 & $0.000^{* *}$ \\
\hline Step 2: CPT omissions & 0.21 & 0.04 & $0.012 *$ & Step 2: CPT omissions & 0.17 & 0.03 & $0.028^{*}$ \\
\hline or Step 1: IQ & 0.14 & 0.02 & 0.098 & or Step 1: Group & -0.35 & 0.12 & $0.000 * *$ \\
\hline Step 2: CPT commissions & 0.18 & 0.03 & $0.029 *$ & Step 2: CPT commissions & 0.16 & 0.02 & $0.045^{*}$ \\
\hline \multicolumn{8}{|l|}{ Columbia Impairment Scale } \\
\hline Step 1: IQ & 0.35 & 0.12 & $0.000 * *$ & Step 1: Group & -0.53 & 0.28 & $0.000 * *$ \\
\hline Step 2: ROCF EPS & 0.20 & 0.04 & $0.004 *$ & Step 2: ROCF EPS & 0.12 & 0.01 & 0.055 \\
\hline or Step 1: IQ & 0.33 & 0.11 & $0.000^{* *}$ & or Step 1: Group & -0.55 & 0.31 & $0.000^{* *}$ \\
\hline Step 2: CPT omissions & 0.12 & 0.01 & 0.081 & Step 2: CPT omissions & 0.06 & 0.00 & 0.335 \\
\hline or Step 1: IQ & 0.33 & 0.11 & $0.000 * *$ & or Step 1: Group & -0.55 & 0.31 & $0.000 * *$ \\
\hline Step 2: CPT commissions & 0.09 & 0.01 & 0.173 & Step 2: CPT commissions & 0.06 & 0.00 & 0.356 \\
\hline
\end{tabular}

$I Q$ intelligence quotient, WIAT Wechsler Individual Achievement Test, ROCF Rey-Osterrieth Complex Figure (EPS error proportion score), CPT Continuous Performance Test

$* p<0.05 ; * *<<0.01$ 
Table 4 Binary Logistic Regression Analyses for Baseline EF Variables and Follow-up PA Variables

\begin{tabular}{|c|c|c|c|c|c|c|c|c|c|}
\hline & Wald & OR & Sig. & $95 \% \mathrm{CI}$ & & Wald & OR & Sig. & $95 \% \mathrm{CI}$ \\
\hline \multicolumn{10}{|l|}{ Peer Acceptance PA } \\
\hline Step 1: IQ & 2.56 & 1.02 & 0.110 & $1.00-1.04$ & Step 1: Group & 14.35 & 0.23 & $0.000^{* *}$ & $0.11-0.50$ \\
\hline Step 2: ROCF Organization & 3.68 & 1.11 & 0.055 & $0.99-1.23$ & Step 2: ROCF Organization & 2.72 & 1.09 & 0.099 & $0.98-1.21$ \\
\hline or Step 1: IQ & 2.51 & 1.02 & 0.113 & $1.00-1.04$ & or Step 1: Group & 14.59 & 0.23 & $0.000^{* *}$ & $0.11-.49$ \\
\hline Step 2: ROCF EPS & 11.98 & 23.91 & $0.001 * *$ & $3.96-144.28$ & Step 2: ROCF EPS & 7.55 & 12.40 & $0.006^{* *}$ & $2.06-74.73$ \\
\hline or Step 1: IQ & 1.83 & 1.02 & 0.176 & $0.99-1.04$ & or Step 1: Group & 17.45 & 0.18 & $0.000 * *$ & $0.08-0.40$ \\
\hline Step 2: CPT omissions & 9.57 & 1.05 & $0.002 *$ & $1.02-1.08$ & Step 2: CPT omissions & 6.29 & 1.04 & $0.012^{*}$ & $1.01-1.07$ \\
\hline or Step 1: IQ & 1.83 & 1.02 & 0.176 & $0.99-1.04$ & or Step 1: Group & 17.45 & 0.18 & $0.000^{* *}$ & $0.08-0.40$ \\
\hline Step 2: CPT commissions & 4.94 & 1.02 & $0.026^{*}$ & $1.01-1.04$ & Step 2: CPT commissions & 4.20 & 1.02 & $0.041 *$ & $1.01-1.04$ \\
\hline \multicolumn{10}{|l|}{ Academic Achievement PA } \\
\hline Step 1: IQ & 40.59 & 1.13 & $0.000 * *$ & $1.09-1.18$ & Step 1: Group & 18.17 & 0.07 & $0.000 * *$ & $0.02-0.24$ \\
\hline Step 2: ROCF EPS & 0.32 & 1.87 & 0.571 & $0.21-16.38$ & Step 2: ROCF EPS & 1.67 & 3.48 & 0.197 & $0.52-23.06$ \\
\hline or Step 1: IQ & 36.10 & 1.13 & $0.000 * *$ & $1.09-1.18$ & or Step 1: Group & 15.44 & 0.05 & $0.000^{* *}$ & $0.01-0.23$ \\
\hline Step 2: CPT omissions & 8.27 & 0.90 & $0.004 * *$ & $0.83-0.97$ & Step 2: CPT omissions & 4.77 & 0.95 & $0.029 *$ & $0.90-0.99$ \\
\hline \multicolumn{10}{|l|}{ Social Skills PA } \\
\hline Step 1: IQ & 6.70 & 1.03 & $0.010 * *$ & $1.01-1.05$ & Step 1: Group & 29.86 & 0.15 & $0.000^{* *}$ & $0.08-0.30$ \\
\hline Step 2: ROCF EPS & 1.70 & 2.91 & 0.193 & $0.58-14.46$ & Step 2: ROCF EPS & 0.05 & 1.22 & 0.818 & $0.23-6.60$ \\
\hline or Step 1: IQ & 6.15 & 1.03 & $0.013 *$ & $1.01-1.05$ & or Step 1: Group & 31.46 & 0.14 & $0.000^{* *}$ & $0.07-0.27$ \\
\hline Step 2: CPT commissions & 5.03 & 1.04 & $0.025^{*}$ & $1.01-1.07$ & Step 2: CPT commissions & 2.37 & 1.03 & 0.123 & $0.99-1.06$ \\
\hline
\end{tabular}

OR odds ratio, $I Q$ intelligence quotient, $R O C F$ Rey-Osterrieth Complex Figure (EPS error proportion score), CPT Continuous Performance Test $* p<0.05 ; * * p<0.01$

IQ, no statistically significant relationships were found in the comparison group, but one statistically significant relationship was discovered in the ADHD group: Baseline ROCF EPS significantly predicted follow-up CIS in the ADHD group, $b=0.19, t(120)=2.09, p=0.039$, but not in the comparison group, $\mathrm{b}=-0.08, t(76)=-0.73, p=0.467$. A Fisher $r$-to- $z$ transformation revealed that the partial correlations between baseline ROCF EPS and follow-up CIS significantly differed between the ADHD and comparison groups $(z=1.87, p<$ $0.05)$. Thus, better baseline ROCF performance predicted higher follow-up CIS scores for the girls with ADHD but not for the comparison girls.

\section{Discussion}

Our chief aim was to determine whether, in girls with and without ADHD, childhood levels of EF could predict adolescent functional outcomes across the domains of academic achievement, social functioning, and global functioning. By using well-established measures of EF as well as several well-validated criterion measures in domains of central importance to healthy functioning, we found that, under tight statistical control, childhood EF predicted adolescent outcomes in academic achievement (including WIAT Math scores and a dichotomous measure of PA in academic achievement) and social functioning (both teacher-appraised peer status and a dichotomous measure of PA in social skills) across the entire sample. Although group status did not emerge as a statistically significant moderator of any associations, separate examination of predictive associations in the ADHD and comparison groups revealed that childhood EF predicted adolescent global functioning in girls with ADHD but not in comparison girls, independent of IQ. In all cases of significant associations, better EF scores at baseline predicted better adolescent outcomes.

In terms of academic achievement, childhood EF as measured by the ROCF EPS and CPT omissions predicted academic achievement outcomes in adolescence across our whole sample (WIAT Math scores, academic achievement PA), with group status controlled. These findings are in line with research showing that children with ADHD and EF deficits have lower academic achievement than those without EF deficits or than non-ADHD comparison youth (Biederman et al. 2004). However, because these predictive associations were significant in our whole sample (with group status failing to moderate this association), our findings indicate that poorer EF scores predict lower academic achievement (namely math achievement) in all girls. This finding is important because it suggests that intervening with EFs may be one possible way to preclude academic difficulties not just in children with specific psychiatric diagnoses, like ADHD, but in typically developing children as well. 
None of our baseline EF measures uniquely predicted WIAT Reading scores. One reason for this negative finding could be that word reading (the skill tapped by the WIAT Basic Reading composite score) becomes automatic for most youth during elementary school (Chall 1983), whereas math skills are cumulatively learned over time, throughout middle and high school. The association between baseline ROCF EPS and follow-up WIAT Math scores could also be explained by the strong visuospatial nature of both of these measures.

In terms of social functioning across our entire sample, childhood EF as measured by CPT omissions and CPT commissions predicted teacher-rated social preference in adolescence across our whole sample when controlling for group status. Additionally, the ROCF EPS joined the CPT indicators in predicting peer acceptance PA across the whole sample. These whole-sample predictions suggest the importance of the abilities to both inhibit one's behavior (i.e., be less impulsive) and pay attention to relationships and social interactions with respect to peer regard. These results are in contrast to other studies finding no such linkages (e.g., Biederman et al. 2004) but are consistent with research showing that $\mathrm{EF}$ impairment has a negative impact on peer acceptance in girls with ADHD (Diamantopoulou et al. 2007) and on social relationships in individuals with autism spectrum disorders (Gilotty et al. 2002). Diamantopoulou et al. (2007) also found that the association between EF deficits and peer acceptance was more pronounced in girls with ADHD than in boys with ADHD (who exhibited equivalent symptomatology), which may be related to (a) the gender-atypical nature of ADHD symptoms in girls and/or (b) girls' penchant for engagement in relational aggression (Zalecki and Hinshaw 2004). Overall, our findings within the realm of social functioning indicate that interventions targeting EF could potentially prevent social difficulties in girls, particularly those with ADHD, who are at high risk for having social problems (e.g. Hinshaw and Blachman 2005).

For global functioning across the whole sample, childhood EF did not predict the global functioning measure (CIS) in adolescence. However, because the correlation between childhood ROCF EPS and adolescent CIS was statistically significant, we explored this association further via separate regression analyses in each group (ADHD vs. comparison). These analyses showed that childhood ROCF EPS predicted adolescent CIS when controlling for IQ in the ADHD group, but not in the comparison group. Although previous studies have not found associations between EF deficits and global functioning in those with ADHD (Biederman et al. 2004), our findings are corroborated by research into the role of EFs in functional impairments in non-ADHD populations (e.g., Alzheimer's disease), which reveal that executive dysfunction can explain a large proportion of the variance in activities of daily living, a global measure of functional abilities (Boyle et al. 2003). Additionally, a recent study found that EFs moderated the association between temperament and global functioning in a sample of typically developing preschoolers (Healy et al., in press). Although such associations were not explored in the present study, combining these findings with ours suggests the importance of intact EFs in terms of global outcomes. In all, this finding amplifies our earlier domain-specific results with respect to academic and social functioning, suggesting strongly that deficits in $\mathrm{EF}$ in childhood have a wide-ranging impact on later functioning for girls with ADHD. These findings also reemphasize the potential importance of treatments that target EFs in order to prevent the development of difficulties across many life domains.

It is of interest that the EPS method of scoring the ROCF was a stronger predictor of functional outcomes than was the more traditionally used organization score. This pattern of results may seem counterintuitive, as the organization score taps overall perceptual conformation and has been shown to be developmentally sensitive (Bernstein and Waber 1996). Yet our own previous work has shown that the EPS method was able to distinguish between girls with ADHD and comparison girls whereas the organization method was not (Sami et al. 2003). Combining these findings with the results from the present study suggests that the EPS is better at differentiating girls with ADHD from comparison girls than the Developmental Scoring System's organization score and may be more useful in terms of predicting adolescent outcomes. It may be that the EPS method of scoring the ROCF allows for the measurement of a large number of EFs within one scoring system (e.g., planning, working memory, inhibitory control, organization, reasoning). Therefore, the EPS may be better able to tap the complex nature of the ROCF as a global EF measure by assessing multiple aspects of $\mathrm{EF}$ at once.

It is also of interest that the CUL did not predict of any of the functional domains assessed. The reason for the CUL's poor predictive power is unclear. It is possible that this task requires less effortful control than initially thought, and may be too simple to require extensive EF. It could also be that it requires more visuospatial abilities than executive abilities, although given that the ROCF has a strong visuospatial component, this would seem a less likely possibility.

Our findings highlight the non-specificity of EF deficits, given that most of the associations between baseline $\mathrm{EF}$ measures and follow-up functional measures were found across the entire sample and not solely in the ADHD group. Although other studies have not found that EF deficits affect functional outcomes in control participants (Biederman et al. 2004), our findings are in line with work by Bull et al. (2008), who found an association between EF and learning in general in a longitudinal study of non-clinical preschoolers. As described earlier, EFs include such abilities 
as planning, organizing tasks and behavior, attentional and inhibitory control, reasoning, and working memory (Pennington and Ozonoff 1996; Tranel et al. 1994). If such aspects of EF are impaired during childhood, it seems likely that academics and social abilities would be significantly and cumulatively influenced over time, regardless of one's psychiatric diagnosis (or lack thereof). For example, imagine a child who has difficulty organizing her schoolwork, keeping the teacher's instructions or a conversation with a friend in working memory, and reasoning. Without intervention to improve such deficits, a pattern of performance would be likely to develop resulting in concurrent and future problems in other domains relying on EF abilities. She may lose assignments, miss instructions, and have poor relations with her peers, resulting in lower grades and fewer positive social interactions. Given our findings, it seems likely that this detrimental effect of EF deficits on later performance is present in all girls exhibiting EF difficulties, regardless of psychiatric diagnosis.

Limitations include the fact that the baseline neuropsychological battery was formulated approximately 15 years ago; including other newly developed measures of EF may well have been useful. Additionally, the degree to which these predictive associations would appear in a representative community sample of girls with ADHD is not clear, as our sample was derived clinically, ascertained from medical, mental health, and school sources as well as advertisements (see Hinshaw 2002). Although there are other outcome domains of importance, such as substance use, this outcome will become more sensitive as our sample ages into young adulthood. It is also possible that the restricted range in the comparison sample contributed to our finding of significant EF-global functioning associations in the ADHD group but not in the comparison group. Additionally, although the associations we found were statistically significant and robust to statistical control of key covariates, there are multiple other factors that contribute to adolescent outcomes aside from EF. Certainly genetic, psychosocial, family, and other environmental factors play significant roles. Indeed, it is likely a complex interaction among these variables - including neuropsychological variables - that is the best predictor of adolescent outcomes. This is an important question for future research. Finally, because our sample is entirely female, generalizability to boys cannot be assumed.

Overall, our findings are consistent with research that has shown that EF deficits predict academic difficulties (Biederman et al. 2004) and that EFs are involved in social abilities (Diamantopoulou et al. 2007), potentially serving as precursors to successful social interactions by bolstering certain underlying constructs such as ToM (Hughes and Ensor 2007; McEvoy et al. 1992). Because approximately half of individuals with ADHD display distinct EF deficits
(Nigg et al. 2005), understanding the potential consequences of these deficits is important, particularly because they appear to persist through adolescence (Hinshaw et al. 2007). Additionally, because our findings suggest that EF predicts various outcome domains across our entire sample (including comparison girls), it seems important to evaluate EF deficits in seemingly typically developing children in addition to those at high-risk for EF deficits. These results again emphasize the non-specificity of EF deficits and their effects.

Our findings may help to consider preventive interventions across other samples displaying EF impairments, such as children with autism or those from low socioeconomic backgrounds (see Kishiyama et al. 2009). Relevant interventions may include computerized EF training and organizational skills training. Indeed, interventions that specifically target improving EFs in preschool children have shown promising results (Diamond et al. 2007). This intervention is particularly interesting given its integration into classroom settings. Other computerized interventions focusing on specific aspects of EF (namely, working memory) have also been shown to be effective in reducing symptom severity and improving working memory in children with ADHD (Klingberg et al. 2002; Klingberg et al. 2005); one study showed increases in prefrontal and parietal activity during working memory tasks after treatment (Olesen et al. 2003). Although this working memory training paradigm is indeed promising, it will be important for researchers other than the developer to replicate such results. Also, our results provide initial support for screening children for EF deficits to initiate preventive measures, although far more work on psychometrics and predictive validity is needed before such an endeavor can become a reality. Additional longitudinal work investigating the predictive associations between early EF deficits and functional outcomes across a variety of populations displaying significant $\mathrm{EF}$ difficulties is of high priority, as are data regarding the effectiveness of $\mathrm{EF}$ training programs for children with distinct EF deficits.

Open Access This article is distributed under the terms of the Creative Commons Attribution Noncommercial License which permits any noncommercial use, distribution, and reproduction in any medium, provided the original author(s) and source are credited.

\section{References}

Barkley, R. A. (1997). Behavioral inhibition, sustained attention, and executive functions: Constructing a unifying theory of ADHD. Psychological Bulletin, 121, 65-94.

Barkley, R. A. (2006). Attention deficit hyperactivity disorder: A handbook for diagnosis and treatment (3rd ed.). New York: Guilford.

Barry, T. D., Lyman, R. D., \& Klinger, L. G. (2002). Academic underachievement and attention- deficit/hyperactivity disorder: 
the negative impact of symptom severity on school performance. Journal of School Psychology, 40, 259-283.

Benson, D. F. (1991). The role of frontal dysfunction in attention deficit hyperactivity disorder. Journal of Child Neurology, 6, S9-S12.

Bernstein, J. H., \& Waber, D. P. (1996). Developmental scoring system for the Rey-Osterrieth Complex Figure. Odessa: Psychological Assessment Resources.

Biederman, J., Monuteaux, M., Seidman, L., Doyle, A. E., Mick, E., Wilens, T., et al. (2004). Impact of executive function deficits and ADHD on academic outcomes in children. Journal of Consulting and Clinical Psychology, 72, 757-766.

Bird, H. R., Shaffer, D., Fisher, P., \& Gould, M. S. (1993). The Columbia Impairment Scale (CIS): pilot findings on a measure of global impairment for children and adolescents. International Journal of Methods in Psychiatric Research, 3, 167-176.

Boyle, P. A., Malloy, P. F., Salloway, S., Cahn-Weiner, D. A., Cohen, R., \& Cummings, J. L. (2003). Executive dysfunction and apathy predict functional impairment in Alzheimer's disease. American Journal of Geriatric Psychiatry, 11, 214-221.

Bull, R., Espy, K. A., \& Wiebe, S. A. (2008). Short-term memory, working memory, and executive functioning in preschoolers: longitudinal predictors of mathematical achievement at age 7 years. Developmental Neuropsychology, 33, 205-228.

Cabeza, R., \& Nyberg, L. (1997). Imaging cognition: an empirical review of PET studies with normal subjects. Journal of Cognitive Neuroscience, 9, 1-26.

Carte, E. T., Nigg, J. T., \& Hinshaw, S. P. (1996). Neuropsychological functioning, motor speed, and language processing in boys with and without ADHD. Journal of Abnormal Child Psychology, 24, 481-498.

Chall, J. S. (1983). Stages in reading development. New York: McGraw-Hill.

Clark, C., Prior, M., \& Kinsella, G. (2002). The relationship between executive function abilities, adaptive behaviour, and academic achievement in children with externalising behaviour problems. Journal of Child Psychology and Psychiatry, 43, 785-796.

Coghill, D., Nigg, J., Rothenberger, A., Sonuga-Barke, E., \& Tannock, R. (2005). Whither causal models in the neuroscience of ADHD? Developmental Science, 8, 105-114.

Cohen, J. (1988). Statistical power analysis for the behavioral sciences. Mahwah: Erlbaum.

Conners, C. K. (1995). Conners' continuous performance test computer program: User's manual. Toronto: Multi-Health Systems.

Diamantopoulou, S., Rydell, A., Thorell, L. B., \& Bohlin, G. (2007). Impact of executive functioning and symptoms of attention deficit hyperactivity disorder on children's peer relations and school performance. Developmental Neuropsychology, 32, 521-542.

Diamond, A., Barnett, W. S., Thomas, J., \& Munro, S. (2007). Preschool program improves cognitive control. Science, 318, 1387-1388.

Dishion, T. (1990). The peer context of troublesome child and adolescent behavior. In P. E. Leone (Ed.), Understanding troubled and troubling youth (pp. 128-153). Thousand Oaks: Sage.

Fischer, M., Barkley, R., Smallish, L., \& Fletcher, K. (2005). Executive functioning in hyperactive children as young adults: attention, inhibition, response perseveration, and the impact of comorbidity. Developmental Neuropsychology, 27, 107-133.

Gilotty, L., Kenworthy, L., Sirian, L., Black, D. O., \& Wagner, A. E. (2002). Adaptive skills and executive function in autism spectrum disorders. Child Neuropsychology, 4, 241-248.

Halperin, J. M., \& Schulz, K. P. (2006). Revisiting the role of the prefrontal cortex in the pathopysiology of attention-deficit/ hyperactivity disorder. Psychological Bulletin, 132, 560-581.

Harlow, J. M. (1848). Passage of an iron rod through the head. Boston Medical and Surgical Journal, 39, 389-393.

Healy, D. M., Brodzinsky, L. K., Bernstein, M., Rabinovitz, B., \& Halperin, J. M. (in press). Moderating effects of neurocognitive abilities on the relationship between temperament and global functioning. Child Neuropsychology.

Hinshaw, S. P. (2002). Preadolescent girls with attention-deficit/ hyperactivity disorder: I. Background characteristics, comorbidity, cognitive and social functioning, and parenting practices. Journal of Consulting and Clinical Psychology, 70, 1086-1098.

Hinshaw, S. P., \& Blachman, D. R. (2005). Attention-deficit/ hyperactivity disorder. In D. Bell-Dolan, S. Foster \& E. J. Mash (Eds.), Handbook of behavioral and emotional problems in girls (pp. 117-147). New York: Kluwer Academic/Plenum.

Hinshaw, S. P., Carte, E. T., Sami, N., Treuting, J. J., \& Zupan, B. A. (2002). Preadolescent girls with attention-deficit/hyperactivity disorder: II. Neuropsychological performance in relation to subtypes and individual classification. Journal of Consulting and Clinical Psychology, 70, 1099-1111.

Hinshaw, S. P., Owens, E. B., Sami, N., \& Fargeon, S. (2006). Prospective follow-up of girls with attention-deficit/hyperactivity disorder into adolescence: evidence for continuing cross- domain impairment. Journal of Consulting and Clinical Psychology, 74, 489-499.

Hinshaw, S. P., Carte, E. T., Fan, C., Jassy, J. S., \& Owens, E. B. (2007). Neuropsychological functioning of girls with attention-deficit/ hyperactivity disorder followed prospectively into adolescence: evidence for continuing deficits? Neuropsychology, 21, 263-273.

Hughes, C., \& Ensor, R. (2007). Executive function and theory of mind: predictive relations from ages 2 to 4 . Developmental Psychology, 43, 1447-14591.

Jurado, M. B., \& Rosselli, M. (2007). The elusive nature of executive functions: a review of our current understanding. Neuropsychology Review, 17, 213-233.

Kishiyama, M. M., Boyce, W. T., Jimenez, A. M., Perry, L. M., \& Knight, R. T. (2009). Socioeconomic disparities affect prefrontal function in children. Journal of Cognitive Neuroscience.

Klingberg, T., Forssberg, H., \& Westerberg, H. (2002). Training of working memory in children with ADHD. Journal of Clinical Experimental Neuropsychology, 24, 781-791.

Klingberg, T., Dahlstrom, K., Fernell, E., Gillberg, C. G., Gustafsson, P., Johnson, M., et al. (2005). Computerized training of working memory in children with ADHD-A randomized, controlled trial. Journal of the American Academy of Child and Adolescent Psychiatry, 44, 177-186.

Lahey, B. B., Pelham, W. E., Loney, J., Kipp, H., Ehrhardt, A., Lee, S. S., et al. (2004). Three- year predictive validity of DSM-IV attentiondeficit hyperactivity disorder in children diagnosed at 4-6 years of age. American Journal of Psychiatry, 161, 2014-2020.

Lee, S. S., Lahey, B. B., Owens, E. B., \& Hinshaw, S. P. (2008). Few preschool boys and girls with ADHD are well adjusted during adolescence. Journal of Abnormal Child Psychology.

Lezak, M. (1995). Neuropsychological assessment (3rd ed.). New York: Oxford University Press.

Massetti, G. M., Lahey, B. B., Pelham, W. E., Loney, J., Ehrhardt, A., Lee, S. S., et al. (2007). Academic achievement over 8 years among children who met modified criteria for attention- deficit/ hyperactivity disorder at 4-6 years of age. Journal of Abnormal Child Psychology, 36, 399-410.

McClelland, G. H., \& Judd, C. M. (1993). Statistical difficulties in detecting interactions and moderator effects. Psychological Bulletin, 114, 376-390.

McEvoy, R. E., Rogers, S. J., \& Pennington, B. F. (1992). Executive function and social communication deficits in young autistic children. Journal of Child Psychology and Psychiatry, 34, 563-578.

Miller, G. A., \& Chapman, J. P. (2001). Misunderstanding analysis of covariance. Journal of Abnormal Psychology, 110, 40-48.

MTA Cooperative Group. (1999). Fourteen-month randomized clinical trial of treatment strategies for attention-deficit hyperactivity disorder. Archives of General Psychiatry, 56, 1073-1086. 
Nigg, J. T., Hinshaw, S. P., Carte, E. T., \& Treuting, J. J. (1998). Neuropsychological correlates of childhood attention-deficit/ hyperactivity disorder: Explainable by comorbid disruptive behavior or reading problems? Journal of Abnormal Psychology, 107, 468-480.

Nigg, J. T., Blaskey, L. G., Huang-Pollock, C. L., \& Rappley, M. D. (2002). Neuropsychological executive functions and DSM-IV ADHD subtypes. Journal of the American Academy of Child \& Adolescent Psychiatry, 41, 59-66.

Nigg, J. T., Willcutt, E. G., Doyle, A. E., \& Sonuga-Barke, E. J. S. (2005). Causal heterogeneity in attention-deficit/hyperactivity disorder: do we need neuropsychologically impaired subtypes? Biological Psychiatry, 57, 1224-1230.

Ogg, R. J., Zou, P., Allen, D. N., Hutchins, S. B., Dutkiewicz, R. M., \& Mulhern, R. K. (2008). Neural correlates of a clinical continuous performance test. Magnetic Resonance Imaging, 26, 504-512.

Olesen, P. J., Westerberg, H., \& Klingberg, T. (2003). Increased prefrontal and parietal activity after training of working memory. Nature Neuroscience, 7, 75-79.

Osmon, D. C. (1996). Understanding symptoms of medial frontal lobe disorder: a clinical case study. Journal of Clinical Psychology in Medical Settings, 3, 23-29.

Osterrieth, P. A. (1944). Le test de copie d'une figure complex [A test of copying a complex figure]. Archives de Psychologie, 30, 206-256.

Owens, E. B., Hinshaw, S. P., Lee, S. S., \& Lahey, B. B. (2009). Few girls with childhood attention-deficit/hyperactivity disorder show positive adjustment during adolescence. Journal of Clinical Child and Adolescent Psychology, 38, 1-12.

Pennington, B. F., \& Ozonoff, S. (1996). Executive functions and developmental psychopathology. Journal of Child Psychology and Psychiatry, 37, 51-87.

Rourke, B. P., \& Orr, R. R. (1977). Prediction of reading and spelling performance of normal and retarded readers: a 4-year follow-up. Journal of Abnormal Child Psychology, 5, 9-20.

Sami, N., Carte, E. T., Hinshaw, S. P., \& Zupan, B. A. (2003). Performance of girls with ADHD and comparison girls on the Rey-Osterrieth Complex Figure: evidence for executive processing deficits. Child Neuropsychology, 9, 237-254.

Sandstrom, M. J., \& Cillessen, A. H. N. (2003). Sociometric status and children's peer experiences: use of the daily diary method. Merrill-Palmer Quarterly, 49, 427-452.

Seidman, L. J., Biederman, J., Monuteaux, M. C., Valera, E., Doyle, A. E., \& Faraone, S. V. (2005a). Impact of gender and age on executive functioning: do girls and boys with and without attention deficit hyperactivity disorder differ neuropsychologi- cally in preteen and teenage years? Developmental Neuropsychology, 27, 79-105.

Seidman, L. J., Valera, E. M., \& Makris, N. (2005b). Structural brain imaging of attention- deficit/hyperactivity disorder. Biological Psychiatry, 57, 1263-1272.

Seidman, L. J., Biederman, J., Valera, E. M., Monuteaux, M. C., Doyle, A. E., \& Faraone, S. V. (2006). Neuropsychological functioning in girls with attention-deficit/hyperactivity disorder with and without learning disabilities. Neuropsychology, 20, 166-177.

Shaffer, D., Fisher, P., Lucas, C. P., Dulcan, M. K., \& Schwab-Stone, M. E. (2000). NIMH Diagnostic Interview Schedule for Children, Version IV (NIMH DISC-IV): description, differences from previous versions, and reliability of some common diagnoses. Journal of the American Academy of Child and Adolescent Psychiatry, 39, 28-38.

Somerville, J., Tremont, G., \& Stern, R. A. (2000). The boston qualitative scoring system as a measure of executive functioning in Rey-Osterrieth Complex Figure performance. Journal of Clinical and Experimental Neuropsychology, 5, 613-621.

Tranel, D., Anderson, S. W., \& Benton, A. (1994). Development of the concept of "executive function" and its relationship to the frontal lobes. In F. Boller \& J. Grafman (Eds.), Handbook of neuropsychology (Vol. 9, pp. 125-148). New York: Elsevier.

Troyer, A. K., \& Wishart, H. A. (1997). A comparison of qualitative scoring systems for the Rey Osterrieth complex figure test. The Clinical Neuropsychologist, 4, 381-390.

Waber, D. P., \& Holmes, J. M. (1985). Assessing children's copy productions of the Rey-Osterrieth Complex Figure. Journal of Clinical and Experimental Neuropsychology, 7, 264-280.

Watanabe, K., Ogino, T., Nakano, K., Hattori, J., Kado, Y., Sanada, S., et al. (2005). The Rey-Osterrieth Complex Figure as a measure of executive function in childhood. Brain and Development, 27, $564-569$.

Wechsler, D. (1991). Manual for the Wechsler intelligence scale for children (3rd ed.). New York: Psychological Corporation/Harcourt Brace.

Wechsler, D. (1992). Wechsler individual achievement test. New York: Psychological Corporation.

Willcutt, E. G., Doyle, A. E., Nigg, J. T., Faraone, S. V., \& Pennington, B. F. (2005). Validity of the executive function theory of attention-deficit/hyperactivity disorder: a meta-analytic review. Biological Psychiatry, 57, 1336-1346.

Zalecki, C., \& Hinshaw, S. P. (2004). Overt and relational aggression in girls with attention-deficit hyperactivity disorder. Journal of Clinical Child and Adolescent Psychology, 33, 131-143. 